\title{
Optimal ATN biomarkers and their role in predicting cognitive progress of mild cognitive impairment
}

\author{
Rao Song \\ The Second Affiliated Hospital of Chongqing Medical University \\ Xiaojia Wu \\ The Second Affiliated Hospital of Chongqing Medical University \\ Huan Liu \\ GE Healthcare \\ Dajing Guo \\ The Second Affiliated Hospital of Chongqing Medical University \\ Lin Tang \\ The Second Affiliated Hospital of Chongqing Medical University \\ Wei Zhang \\ The Second Affiliated Hospital of Chongqing Medical University \\ Junbang Feng \\ The Second Affiliated Hospital of Chongqing Medical University \\ Chuanming Li ( $\nabla$ lichuanming@hospital.cqmu.edu.cn ) \\ The Second Affiliated Hospital of Chongqing Medical University
}

\section{Research}

Keywords: Alzheimer's disease, Biomarker, Amyloid, Tau, Neurodegeneration, Mild cognitive impairment, Radiomics

Posted Date: February 18th, 2021

DOI: https://doi.org/10.21203/rs.3.rs-201708/v1

License: @) (7) This work is licensed under a Creative Commons Attribution 4.0 International License. Read Full License 


\section{Abstract \\ Background}

It is of great significance to investigate the optimal cerebrospinal fluid (CSF) and magnetic resonance image biomarkers of ATN system and clarify their predictive value in cognitive progression of mild cognitive impairment individuals ( $\mathrm{MCl})$.

\section{Methods}

147 healthy control $(\mathrm{HC}), 197$ patients with $\mathrm{MCl}$, and 128 patients with Alzheimer' Disease (AD) were included from the ADNI database. All MCl patients were

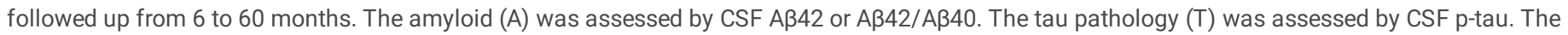
neurodegeneration $(\mathrm{N})$ was assessed by radiomics of the whole brain MRI or by CSF t-tau. Biomarkers with larger area under the receiver operating characteristic curve (AUC) in the discrimination of $A D$ and $\mathrm{HC}$ were considered to be the optimal biomarkers. The conversion rates of different ATN profiles in $\mathrm{MCl}$ subjects during follow-up were analyzed using Kaplan-Meier estimates and compared using the Log-rank test.

\section{Results}

The CSF A 42 and the radiomics signature (AUC 0.822 and 0.998 , respectively) were identified as the optimal $A$ and $N$ biomarkers, respectively. For MCI patients of the Alzheimer continuum, there was no significant difference in the progression rate of $A+T-N-$ and $A-T-N-p r o f i l e s(p>0.05)$. The $A+T+N-$, $\mathrm{A}+\mathrm{T}-\mathrm{N}+$ profiles had a significant higher progression rate than that of the $\mathrm{A}+\mathrm{T}-\mathrm{N}-$ patients (all $p<0.05)$. For $\mathrm{MCl}$ of the suspected non-AD pathophysiology (SNAP), patients with the $\mathrm{A}-\mathrm{T}-\mathrm{N}+$ profile $(p<0.05)$ showed significant higher progression rate than $\mathrm{A}-\mathrm{T}-\mathrm{N}-$ profile. There was no significant difference in the progression rate of $A-T+N-$ and $A-T-N-$ profiles $(p>0.05)$.

\section{Discussion}

We proposed a new radiomics method to assess $\mathrm{N}$ accurately and ascertained the optimal $\mathrm{A} / \mathrm{T} / \mathrm{N}$ biomarkers for the discrimination of $\mathrm{HC}$ and $\mathrm{AD}$. For $\mathrm{MCl}$ patients of the Alzheimer continuum, isolated A + was indicator of cognitive stability, while the abnormality of $\mathrm{T}$ and $\mathrm{N}$, respectively or simultaneously, indicated the high risk of progression. For MCl patients of SNAP, isolated $\mathrm{T}+$ indicated the cognitive stability, while the appearance of $\mathrm{N}+$ indicated the high risk of progression.

\section{Background}

Alzheimer's disease (AD) is the leading cause of dementia and the risk increases with age[1]. The impairment of cognitive function and behavior of $A D$ was progressive and unremitting, which needs long-term care and causes huge family economic burden. It is estimated that by 2020 , the total expenditure of all patients with Alzheimer's disease or other dementia will be $\$ 305$ billion[2]. AD shows a distinct pathology associated with the accumulation of amyloid and tau proteins in the brain. In the early stage of the disease, there is no cognitive impairment, but neuropathological changes have emerged. Timely treatment may be effective before the disease reaches an irreversible degenerative state[3, 4]. Based on the changes in neuropathology, the 2018 National Institute of Aging and Alzheimer's Association proposed the amyloid/tau/neurodegeneration (ATN) classification scheme to redefine AD by biomarkers other than clinical symptoms[5]. Individuals can be classified as abnormal (+) or normal (-) for $\mathrm{A}, \mathrm{T}$ and $\mathrm{N}$, resulting in eight different ATN profiles.

Cerebrospinal fluid (CSF) examination or brain imaging (magnetic resonance image [MRI] or positron emission tomography [PET]) could be used to obtain ATN biomarkers. Due to the high cost and radioactivity, the application of PET was limited. Accessible means like CSF and MRI were most widely used in clinical practice. According to ATN system, the aggregated $\beta$-amyloid (A $\beta$ ) (labeled "A") could be reflected by the baseline CSF levels of A $\beta 42$, or A $342 / A \beta 40$ ratio. Neurodegeneration or neuronal injury (labeled " $\mathrm{N}$ ") could be assessed by baseline CSF levels of total tau (t-tau) or brain atrophy on MRI. However, it is not clear which indicators could accurately identify different cognitive states and should be used as the optimal clinical biomarkers. On the other hand, most previous studies only used a single indicator to assess the atrophy of parts of the brain, such as the volume of the hippocampus[6-8], the rating of the medial temporal lobe atrophy[9-11] or the thickness of the cerebral cortex[6, 8]. In fact, the neural damage of AD on the brain includes all the cortex and most of subcortical nucleus. For example, Lehmann and his colleagues have reported decreased cortical thickness in bilateral posterior cingulate gyrus, precuneus, and posterior parietal lobes in AD patients[12]. Subcortical nuclei like putamen, thalamus and basal ganglia also suffered significant atrophy related to cognitive impairment $[13,14]$. Therefore, it is of great significance to comprehensively investigate the structural changes of brain and obtain a sensitive and accurate $\mathrm{N}$ biomarker to further improve the ATN system.

Mild cognitive impairment $(\mathrm{MCl})$, as a transitional state between normal cognition and AD, has always been the focus of attention. It is estimated that about $60 \%$ of $\mathrm{MCl}$ patients will progress to dementia during 3-year follow-up[15, 16], and this rate will increase to $80 \%$ at 4 -year follow-up. Previously, it has been reported that $\mathrm{MCl}$ patients with different $\mathrm{ATN}$ combinations have different cognitive deterioration risks. Compared with $\mathrm{A}-\mathrm{T}-\mathrm{N}-$-, patients with $\mathrm{A}+\mathrm{T}+\mathrm{N}+\mathrm{had}$ a significantly higher risk of $A D$ dementia $(H R=3.54)[17]$. However, the role and predictive value of isolated $A / T / N$ biomarker in cognitive progression are still unclear. In this study, we first analyzed the structural changes of the whole brain with radiomics technology to establish a new method to evaluate $\mathrm{N}$ biomarker, and determined the optimal ATN indicators by distinguishing healthy control (HC) and AD. Then, all MCl patients were divided into different ATN groups and followed up for five years to investigate the role and predict value of isolated A/T/N biomarker in the cognitive progression. 


\section{Methods \\ Participants}

Data used in this study were downloaded from the Alzheimer's Disease Neuroimaging Initiative (ADNI) website (adni.loni.usc.edu). Totally 147 HC (Health controls), 197 patients with $\mathrm{MCl}$ and 128 patients with $\mathrm{AD}$ were included from the ADNI-GO and ADNI-2. AD patients met the criteria made by NINCDS/ADRDA for probable $A D[18,19]$. Participants with $\mathrm{MCl}$ reported a subjective memory concern, but they showed no significant impairment in other cognitive domains, everyday activities were substantially preserved, and there were with no sign of dementia. The $\mathrm{HC}$ subjects showed no signs of depression, $\mathrm{MCl}$ or dementia. All the participants had complete demographic, clinical, laboratory characteristics and MR images of T1WI at the baseline of data collection. The MCI subjects were followed up for 6-60 months, with a follow-up interval of 6-12 months in the first 3 years and 12 months after 3 years. Among them, 100 patients progressed to dementia and other 97 patients remained stable during the follow-up period. Participants who progressed from $\mathrm{MCI}$ to $\mathrm{AD}$ and back to $\mathrm{MCl}$ during the whole observation period were excluded.

\section{Clinical and CSF characteristics}

Clinical and CSF information were directly collected from the ADNI assessment files. Demographic characteristics included age, sex, education level, alcohol abuse, body mass index (BMI), and prevalence of APOE $\varepsilon 4$. Eleven neuropsychological scales were adopted to evaluate the cognitive function at baseline, including the Mini-Mental State Examination (MMSE), the Alzheimer's Disease Assessment Scale-Cognitive subscale (ADAS-Cog), the Clinical Dementia Rating (CDR), the Functional Activities Questionnaire (FAQ), the Geriatric Depression Scale (GDS), the Rey Auditory Verbal Learning Test (RAVLT) and the Animal Fluency Test (AFT). CSF characteristics included the CSF A $42, A \beta 40$, phosphorylated tau ( $p$-tau) and t-tau protein levels.

\section{MRI acquisition and radiomics feature extraction}

Structural MRI was recorded using three-dimensional magnetization prepared rapid gradient echo (3D MPRAGE) sequence or equivalent scanning scheme on 3.0T scanners. Detailed imaging parameters were available from the ADNI website (http://adni.loni.usc.edu/methods/documents/). For features extraction, FreeSurfer 6.0 software (http://surfer.nmr.mgh.harvard.edu/) was used. Briefly, the procedure included motion correction, removal of the skull, Talairach transformation, gray/white matter segmentation, intensity normalization, topology correction, surface deformation, inflation, registration and parcellation. The whole cortex was divided into 148 cortical regions by Destrieux atlas. Indicators including surface area, average thickness, standard deviation of thickness, integrated rectified Gaussian curvature, integrated rectified mean curvature, intrinsic curvature index, folding index, and gray matter volume were obtained from each of the cortical region. Besides, 14 regions were obtained from the subcortical segmentation by Desikan-Killiany atlas, including bilateral thalamus, caudate, putamen, pallidum, hippocampus, amygdala and nucleus accumbens. The volume of each subcortical structure was obtained. Finally, a total of 1198 image features were extracted.

\section{Feature selection and signature construction}

Harmonization in the feature domain was performed before feature selection. Firstly, the abnormal values were replaced by median. Then, the data was standardized to eliminate the influence of dimension and entered into the following analysis. The Mann-Whitney U test and the spearman rank correlation analysis ( $r$ threshold of 0.9 ) were performed used for feature redundancy. The least absolute shrinkage and selection operator (LASSO) regression algorithm with 5-fold cross-validation was used to select features with nonzero coefficients. Stepwise selection based on Akaike Information Criterion was applied to remove features that were not significant. Finally, the most powerful radiomics features were used to construct the radiomics model on the basis of multivariate logistic regression. The radiomics score (rad-score) of each individual was calculated through a linear combination of selected features multiplied by their respective coefficients.

\section{Biomarker evaluation and screening}

Logistic regression leveraging 5 -fold cross-validation were employed to assess the performance of the optimal radiomics features. This method separated the data into 5 subsets randomly and utilized one subset as the validation set and the remaining subsets as the training set. This process was repeated until every subset was utilized once. The area under the curve (AUC), sensitivity, and specificity were applied to assess the performance of the machine learning model for the discrimination between $\mathrm{HC}$ and AD. For each CSF biomarker, receiver operating characteristic (ROC) curves were applied and Youden's index was calculated to determine the optimal cutoff value. Between CSF A 42 and AB42/AB40 ratio, the one with higher AUC was selected to as the optimal biomarker of A. Similarly, between the radiomics model and CSF t-tau, the one with higher AUC was defined as the optimal biomarker of N. CSF p-tau was adopted as the T biomarker.

\section{Statistical Analysis}

The statistical analysis was performed by SPSS 24.0 (Armonk, NY: IBM Corp.). Frequency (percent) and mean (standard deviation) were used to describe categorical variables and normal distribution continuous variables, median (lower quarter, upper quarter) was used to describe non-normal distribution continuous variables. One-way analysis of variance and Kruskal-Wallis were performed for statistical analysis of continuous variables. When a statistically significant overall difference was detected, pairwise comparisons between groups were conducted by Tukey or Nemenyi post hoc analysis for the correction of multiple comparisons. Chi-square test and Fisher exact test were performed for statistical analysis of categorical variables. When a statistically significant overall difference was detected, pairwise comparisons were adjusted by Bonferroni correction. Survival for MCl patients with different ATN profile was estimated using the Kaplan-Meier method, and any differences in survival were evaluated with a stratified log-rank test for overall comparisons and pairwise comparisons.

\section{Results}




\section{Participants characteristics at baseline}

Baseline characteristics of the participants were displayed in Table 1 and Additional file 1. The AD patients showed a lower BMI value than those of the MCI and $\mathrm{HC}$ subjects. There were no significant group differences regarding age, sex and alcohol abuse among the three groups. For the neuropsychological scales, all groups differed significantly between each other regarding MMSE, ADAS-Cog11, ADAS-Cog13, CDR, FAQ, RAVLT immediate, RAVLT learning, RAVLT percent forgetting and AFT (all $p<0.05$ ). The CSF biomarkers were significantly different between $\mathrm{HC}, \mathrm{MCl}$ and $\mathrm{AD}$ groups (all $p<0.05$ ). The $\mathrm{AD}$ group contained the lowest content of $A \beta 42$ and $A \beta 42 / A \beta 40$ ratio and highest content of $p$-tau and t-tau. The APOE $\varepsilon 4$ also varied by different groups (all $p<0.05$ ), with $A D$ group containing the highest number of $A P O E \& 4$ carriers.

Table 1

Baseline clinical characteristics of $\mathrm{HC}, \mathrm{MCl}$ and $\mathrm{AD}$ groups

\begin{tabular}{|c|c|c|c|c|c|c|}
\hline & $H C(n=147)$ & $M C I(n=197)$ & $A D(n=128)$ & $F / \chi^{2}$-value & $P$-value & $P<0.05^{\star}$ \\
\hline \multicolumn{7}{|l|}{ Demographic information } \\
\hline Age, year & $73.66(6.34)$ & $72.23(7.09)$ & 73.68(8.36) & 4.489 & $>0.05$ & \\
\hline Sex, male & 72(49) & 114(58) & $74(58)$ & 3.216 & $>0.05$ & \\
\hline Education, year & $16.59(2.53)$ & $16.20(2.75)$ & 15.48(3.06) & 5.596 & $<0.05$ & $\mathrm{~b}$ \\
\hline Alcohol abuse & $7(5)$ & $9(5)$ & $10(8)$ & 1.797 & $>0.05$ & \\
\hline BMI & $27.21(4.28)$ & $27.82(5.08)$ & $25.89(5.10)$ & 6.204 & $<0.05$ & $b, c$ \\
\hline APOE $\varepsilon 4$ carrier & $40(27)$ & 105(53) & $86(67)$ & 46.329 & $<0.001$ & $a, b, c$ \\
\hline \multicolumn{7}{|l|}{ Neuropsychological scales } \\
\hline MMSE & 29.08(1.15) & 27.68(1.81) & 23.35(2.05) & 277.967 & $<0.001$ & $a, b, c$ \\
\hline ADAS-Cog11 & $5.87(3.10)$ & $10.75(4.78)$ & 20.35(6.92) & 273.185 & $<0.001$ & $a, b, c$ \\
\hline ADAS-Cog13 & $9.05(4.49)$ & $17.32(7.23)$ & $30.60(8.13)$ & 286.127 & $<0.001$ & $a, b, c$ \\
\hline CDR & $0.00(0.00,0.00)$ & $0.50(0.50,0.50)$ & $1.00(0.50,1.00)$ & 173.549 & $<0.001$ & $a, b, c$ \\
\hline FAQ & $0.50(0.50,0.50)$ & $2.00(0.00,5.50)$ & $13.00(8.00,18.00)$ & 289.777 & $<0.001$ & $a, b, c$ \\
\hline GDS & $0.00(0.00,1.00)$ & $2.00(1.00,3.00)$ & $1.00(1.00,2.00)$ & 77.398 & $<0.001$ & $a, b$ \\
\hline RAVLT immediate & $46.22(10.18)$ & $33.42(9.67)$ & $22.52(6.93)$ & 247.506 & $<0.001$ & $a, b, c$ \\
\hline RAVLT learning & $5.90(2.39)$ & $4.07(2.62)$ & $1.82(1.69)$ & 155.672 & $<0.001$ & $a, b, c$ \\
\hline RAVLT forgetting & $3.84(2.67)$ & $4.96(2.41)$ & $4.39(1.56)$ & 20.674 & $<0.001$ & a \\
\hline RAVLT percent forgetting & $36.00(27.32)$ & $65.28(31.12)$ & $88.69(20.06)$ & 173.549 & $<0.001$ & $a, b, c$ \\
\hline $\mathrm{AFT}$ & $21.54(5.43)$ & $16.85(4.98)$ & $12.30(6.15)$ & 115.174 & $<0.001$ & $a, b, c$ \\
\hline
\end{tabular}

\section{Radiomics model construction and performance evaluation}

Through Mann-Whitney U test and spearman analysis, 503 features were obtained from 1198 features. Then, these features were reduced to 46 features with nonzero coefficients by the LASSO method (Fig. 1A and 1B). After Stepwise selection based on Akaike Information Criterion, 15 optimal features were obtained to build the radiomics model. The optimal features and their coefficients are presented in Fig. 1C. The ROC curve of radiomics model was shown in Fig. 1D. The AUC with 5 -fold nested cross-validation was 0.998 (sensitivity 0.969 , specificity 0.973 ).

\section{Optimal biomarkers for ATN classification and the frequency of the ATN profiles among $\mathrm{HC}, \mathrm{MCl}$ and AD}

As shown in Fig. $2 A$ and $2 B$, the AUC of CSF A 42 was 0.822 , which was higher than that of the ratio of CSF AB42 /AB40 ( 0.813 ), and was considered as the optimal A biomarker. Similarly, the radiomics model showed a higher AUC of 0.998 (Fig. 1C) than CSF t-tau (0.795; Fig. 2D) and was chose as the optimal N biomarker. According to the ATN classification scheme, we classified each participant into three binary categories. A+ refers to $A \beta$ pathology (CSF A 42 levels $\leq 952 \mathrm{pg} / \mathrm{mL}$ ), $\mathrm{T}+$ refers to pathologic $\mathrm{p}$-tau (CSF p-tau $>24.38 \mathrm{pg} / \mathrm{mL}$ ), and $\mathrm{N}+$ refers to the neurodegeneration biomarker (rad-score $>0.4561$ ).

The proportion of participants in the Alzheimer's continuum (A+) increased from $23.1 \%$ in $\mathrm{HC}$ to $56.3 \%$ in $\mathrm{MCl}$ and $78.9 \%$ in $\mathrm{AD}$. The opposite trend was found in individuals with normal biomarker profile (A- T-N-), from $52.4 \%$ in $\mathrm{HC}$ to $21.3 \%$ in $\mathrm{MCl}$ and 0 in $\mathrm{AD}$. The prevalence of SNAP (A- with either $\mathrm{T}+$ or $\mathrm{N}+$ ) was consistent across the three groups (HC: $24.5 \%$; MCl: $22.3 \%$; AD: $21.1 \%$ ). Stratifying by cognitive stage, A- T-N- was the most common ATN profile in HC. By 
contrast, $A-T+N+, A+T-N+$ and $A+T+N+$ profiles were the least common profiles in $H C$, accounting for less than $1 \%$. Among $M C l$ individuals, group with the greatest proportion was $A-T-N-(21.3 \%)$, followed by $A+T+N-(19.8 \%)$ and $A+T+N+(19.8 \%)$. The prevalence of $A+T+N+$ was dominant in $A D$, with a high proportion of $64.8 \%$. Besides, no participant showed a biomarker combination of $A-T-N-$ or $A-T-N+$ in $A D$ (Additional file 2 and Additional file 3 ).

\section{Baseline characteristics and 5-year progression rate of different ATN profile in $\mathrm{MCl}$ patients}

Baseline characteristics of each ATN profile in $\mathrm{MCl}$ were showed in Table 2. There were no significant differences in sex, education level, alcohol abuse, BMI, CDR, or GDS scores among ATN profiles. In the Alzheimer continuum ( $A+)$, There was no significant difference in cognitive function between $A+T-N-$ and $A$ $-\mathrm{T}-\mathrm{N}$ - patients. Patients with a profile of $\mathrm{A}+\mathrm{T}+\mathrm{N}+$ had a higher cognition score of ADAS-Cog13 than those in $\mathrm{A}+\mathrm{T}+\mathrm{N}-(p<0.05)$. There was no significant difference in cognitive function between $\mathrm{A}+\mathrm{T}+\mathrm{N}-$ and $\mathrm{A}+\mathrm{T}-\mathrm{N}-$ patients. For SNAP MCl, patients with $\mathrm{A}-\mathrm{T}+\mathrm{N}-$ profile showed similar neurological scale scores as $\mathrm{A}-\mathrm{T}-\mathrm{N}-$. Compared to $\mathrm{A}-\mathrm{T}-\mathrm{N}-$, patients with the $\mathrm{A}-\mathrm{T}+\mathrm{N}+$ profile were older $(\mathrm{A}-\mathrm{T}-\mathrm{N}-: 69.46 \pm 7.04$ years vs $\mathrm{A}-\mathrm{T}+\mathrm{N}+: 76.33 \pm 8.79$ years, $p<$ 0.05 ) and had a worse memory function measured by RAVLT immediate, RAVLT learning and RAVLT percent forgetting (all $p<0.05)$ and a worse global cognition measured by ADAS-Cog $13(p<0.05)$.

Table 2

Baseline clinical characteristics of the 8 ATN profiles in $\mathrm{MCl}$ patients

\begin{tabular}{|c|c|c|c|c|c|c|c|c|}
\hline & \multirow{2}{*}{$\begin{array}{l}\text { Normal } \\
\text { A-T-N- } \\
(n=42)\end{array}$} & \multicolumn{3}{|l|}{ SNAP } & \multicolumn{4}{|c|}{ Alzheimer continuum } \\
\hline & & $\begin{array}{l}A-T+N- \\
(n=18)\end{array}$ & $\begin{array}{l}A-T-N+ \\
(n=13)\end{array}$ & $\begin{array}{l}A-T+N+ \\
(n=13)\end{array}$ & $\begin{array}{l}A+T-N- \\
(n=17)\end{array}$ & $\begin{array}{l}A+T+N- \\
(n=39)\end{array}$ & $\begin{array}{l}A+T-N+ \\
(n=16)\end{array}$ & $\begin{array}{l}A+ \\
(n=\end{array}$ \\
\hline \multicolumn{9}{|c|}{ Demographic information } \\
\hline Age, year & $69.46(7.04)$ & 72.98(8.15) & 73.32(7.02) & 76.33(8.79) & $70.17(6.18)$ & 71.32(7.01) & 74.74(7.47) & 73.9 \\
\hline Sex, male & $21(50)$ & $11(61)$ & $7(54)$ & $6(46)$ & $13(77)$ & $22(56)$ & $13(81)$ & $21(5$ \\
\hline $\begin{array}{l}\text { Education, } \\
\text { year }\end{array}$ & $16.02(2.72)$ & $16.89(2.59)$ & $16.85(2.51)$ & 16.08(1.85) & $16.12(2.62)$ & $16.05(2.77)$ & $16.06(4.07)$ & 16.1 \\
\hline $\begin{array}{l}\text { Alcohol } \\
\text { abuse }\end{array}$ & $1(2)$ & $0(0)$ & $0(0)$ & $1(8)$ & $1(6)$ & $3(8)$ & $0(0)$ & $3(8)$ \\
\hline BMI & $29.4(5.15)$ & $28.01(4.46)$ & $29.87(7.45)$ & $27.36(3.13)$ & $29.43(7.85)$ & 27.11(4.00) & $27.35(5.46)$ & 25.7 \\
\hline $\begin{array}{l}\text { APOE \&4 } \\
\text { carrier }\end{array}$ & $9(21)$ & $8(44)$ & $4(31)$ & $5(39)$ & $8(47)$ & $31(80)$ & $8(50)$ & $32(\varepsilon$ \\
\hline \multicolumn{9}{|c|}{ Neuropsychological scales } \\
\hline MMSE & $28.48(1.64)$ & $27.56(1.62)$ & 28.31(1.93) & $27(1.87)$ & $28.47(1.18)$ & 27.54(1.68) & $27.56(2.03)$ & 26.7 \\
\hline $\begin{array}{l}\text { ADAS- } \\
\text { Cog11 }\end{array}$ & 7.93(3.34) & $8.83(2.77)$ & $11.13(4.97)$ & 13.54(5.38) & $8.41(3.14)$ & 10.53(3.54) & $11.92(5.14)$ & 14.4 \\
\hline $\begin{array}{l}\text { ADAS- } \\
\text { Cog13 }\end{array}$ & 12.07(5.08) & 15.33(4.69) & 17.51(6.94) & $22.15(7.40)$ & 13.06(3.75) & $17.27(5.78)$ & 18.79(7.02) & 23.5 \\
\hline
\end{tabular}

\begin{tabular}{lllllllll} 
CDR & $0.50(0.50,0.50)$ & $0.50(0.50,0.50)$ & $0.50(0.50,0.50)$ & $0.50(0.50,0.50)$ & $0.50(0.50,0.50)$ & $0.50(0.50,0.50)$ & $0.50(0.50,0.50)$ & $0.5 C$ \\
\hline FAQ & $0.00(0.00,2.00)$ & $2.50(0.00,4.50)$ & $0.00(0.00,5.00)$ & $5.00(0.00,6.00)$ & $1.00(0.00,4.50)$ & $1.00(0.00,4.00)$ & $3.50(1.00,9.00)$ & $6.0 C$ \\
\hline GDS & $2.00(1.00,3.00)$ & $1.00(0.00,3.25)$ & $2.00(1.00,2.50)$ & $1.00(0.50,2.00)$ & $2.00(1.00,3.00)$ & $2.00(1.00,3.00)$ & $2.00(1.00,3.00)$ & $1.0 C$ \\
\hline $\begin{array}{l}\text { RAVLT } \\
\text { immediate }\end{array}$ & $39.38(11.49)$ & $35.44(9.56)$ & $36.92(6.14)$ & $27.46(7.51)$ & $35.29(9.95)$ & $31.69(6.93)$ & $30.56(6.36)$ & 28.9 \\
\hline $\begin{array}{l}\text { RAVLT } \\
\text { learning }\end{array}$ & $5.26(2.57)$ & $4.67(3.01)$ & $4.31(2.39)$ & $2.62(2.50)$ & $5.12(2.47)$ & $3.77(2.62)$ & $3.38(2.22)$ & 3.05 \\
\hline $\begin{array}{l}\text { RAVLT } \\
\text { forgetting }\end{array}$ & $4.12(2.69)$ & $5.94(2.46)$ & $5.92(2.78)$ & $4.92(2.18)$ & $4.06(2.25)$ & $5.1(1.94)$ & $4.88(2.39)$ & 5.41 \\
\hline $\begin{array}{l}\text { RAVLT } \\
\text { percent } \\
\text { forgetting }\end{array}$ & $46.61(30.82)$ & $71.12(28.32)$ & $64.27(22.92)$ & $79.36(27.19)$ & $45.87(29.12)$ & $68.63(28.08)$ & $66.16(33.19)$ & $83 . C$ \\
\hline \begin{tabular}{l} 
AFT \\
\hline
\end{tabular} & $19.38(5.58)$ & $16.72(3.92)$ & $17.23(4.44)$ & $15.69(3.61)$ & $18.18(6.54)$ & $17.36(3.57)$ & $14.94(5.48)$ & 14.1
\end{tabular}

Data are shown as the mean (SD) or number (\%). Fisher exact test with Bonferroni correction was used for the analysis of categorical variables. Kruskal-Wall used for the analysis of age, BMI and ADAS-Cog11. One-way analysis of variance with Tukey post hoc test was used for the analysis of other continuous var $T-N-v s . A+T+N-; c, A-T-N-v s . A+T+N+; d, A-T-N+v s . A+T+N-; e, A-T-N+v s . A+T+N+; f, A+T-N-v s . A+T+N+; g, A-T-N-v s . A+T-N+; h, t$ $-\mathrm{T}+\mathrm{N}+; \mathrm{j}, \mathrm{A}-\mathrm{T}+\mathrm{N}+\mathrm{vs} . \mathrm{A}+\mathrm{T}-\mathrm{N}-; \mathrm{k}, \mathrm{A}+\mathrm{T}+\mathrm{N}-\mathrm{vs} . \mathrm{A}+\mathrm{T}+\mathrm{N}+; \mathrm{l}, \mathrm{A}+\mathrm{T}+\mathrm{N}-\mathrm{vs} . \mathrm{A}+\mathrm{T}+\mathrm{N}+$.

Abbreviations: SNAP, suspected non-Alzheimer pathology; BMI, body mass index; APOE, apolipoprotein; MMSE, Mini-Mental State Examination; ADAS-Cog, Al Cognitive subscale; CDR, Clinical Dementia Rating; FAQ, Functional Activities Questionnaire; GDS, Geriatric Depression Scale; RAVLT, Rey Auditory Verbal Lear 
During the follow-up period of five years, the progression rate of each ATN profile varied (Additional file 4 and Additional file 5 ). The A $+\mathrm{T}+\mathrm{N}+$ profile and $\mathrm{A}-$ $\mathrm{T}-\mathrm{N}$ - profile showed the highest $(92.3 \%)$ and lowest (11.9\%) progression rate, respectively. The Kaplan-Meier curves assessing the cognitive progression of the different ATN profiles were presented in Fig. 3 and the corresponding log-rank test results were shown in Supplementary Table 3. For the Alzheimer continuum, there was no significant difference in the progression rate of $\mathrm{A}+\mathrm{T}-\mathrm{N}-$ and $\mathrm{A}-\mathrm{T}-\mathrm{N}-$ profiles $(p>0.05)$. The $\mathrm{A}+\mathrm{T}+\mathrm{N}-$ and $\mathrm{A}+\mathrm{T}-\mathrm{N}+$ profiles both had significant higher progression rates than that of the $A+T-N-$ patients (both $p<0.05$ ). The progression rate of the $A+T+N+$ profile was significantly higher than those in $\mathrm{A}+\mathrm{T}+\mathrm{N}-(p<0.001)$ or $\mathrm{A}+\mathrm{T}-\mathrm{N}+$ profile $(p<0.05)$. For $\mathrm{MCl}$ of SNAP, patients with $\mathrm{A}-\mathrm{T}-\mathrm{N}+(p<0.05)$ and $\mathrm{A}-\mathrm{T}+\mathrm{N}+(p<0.001)$ profiles showed significant higher progression rate than $\mathrm{A}-\mathrm{T}-\mathrm{N}-$. There was no significant difference in the progression rate of $\mathrm{A}-\mathrm{T}+\mathrm{N}-$ and $\mathrm{A}-\mathrm{T}-\mathrm{N}-\mathrm{profiles}(p>$ 0.05).

\section{Discussion}

Neurodegeneration is a characteristic of neuropathological changes in AD, which is closely related to symptoms[20]. Brain atrophy can reflect the degree of neurodegeneration and can be detected by magnetic resonance imaging. Most of the previous studies only used a single indicator of the volume or thickness of $A D$ specific regions such as hippocampus and temporal lobe to evaluate $\mathrm{N}$ biomarker[6-11]. Many other important brain areas and features were ignored. As a new discipline, radiomics can extract a large number of high-throughput imaging features from traditional medical images, and use machine learning to establish an artificial intelligence model to improve the accuracy of identification. In recent years, it has been widely used in the diagnosis, classification and prognosis prediction of neurodegenerative diseases such as AD and Parkinson's disease[21-25]. As far as we know, this is the first time that a radiomics method based on magnetic resonance imaging of the whole brain has been used to evaluate the $\mathrm{N}$ biomarker. Our sensitivity and specificity for the discrimination of $\mathrm{HC}$ and $\mathrm{AD}$ were 0.969 and 0.973 , which were higher than hippocampal volume (sensitivity 0.673 , specificity 0.803 ) or brain mean cortical thickness (sensitivity of 0.833 , specificity 0.859 ) reported previously[6]. Different MRI indexes can reflect the brain atrophy from different aspects. The identification accuracy of our combined multiple indicators including cortical thickness, cortical area, cortical curvature and subcortical volume was higher than that of single index. Most selected features were located in the temporal, frontal and parietal cortices. Structural changes in these cortical regions have been reported in patients with $A D$ pathology and have been proven to be associated with disease progression[26-28]. In typical cases of $A D$, abnormalities of amyloid plaques or neurofibrillary tangles usually first appear in regions of temporal lobes and hippocampus, and progressively spread to frontal lobes and other areas of the cortex[29]. Parietal lobe is considered as a multimodal area of cognition. Parietal lobe dysfunction may be one of the causes of cognitive dysfunction in early $\mathrm{AD}[30]$. The volume of the amygdala was also a retained feature for radiomics model establishment. Previous studies have reported that the decrease of amygdala volume is related to cognitive dysfunction and can be used as a marker of dementia severity in AD patients[31, 32]. In this study, we also found that the accuracy of radiomics model was significantly higher than that of CSF t-tau, which should be used as the optimal method to evaluate $\mathrm{N}$. The possible reason is that the brain atrophy on MRI reflects the cumulative loss and damage of nerve cells, while t-tau in cerebrospinal fluid only reflects the damage of neurons at a certain time point. For A biomarker, CSF A 42 was selected as the optimal biomarker because it performed slightly better than $A \beta 42 / A \beta 40$ ratio. The reduction in CSFAB42 in AD have consistently been found to reflect a deposition of the peptide in senile plaques, with lower levels diffusing to the CSF. As a hallmark feature of $A D, C S F A \beta 42$ also have been reported to be useful for discriminating $A D$ from $\mathrm{HC}$ and $M C I[33-35]$.

According to the ATN system, excessive $\beta$-Amyloidosis deposition (A+) is the biomarker of Alzheimer's pathologic change. In this study we found that the frequency of patients with $\mathrm{A}+$ increased with the clinical diagnostic severity, from $23.1 \%$ in $\mathrm{HC}$, via $56.3 \%$ in $\mathrm{MCl}$, and finally yielded the highest rate of $78.9 \%$ in $A D$. This result is consistent with previous studies, although the exact prevalence of the $A+$ profiles varies from study to study[6, 9, 17]. During the 5 -year follow-up period of $\mathrm{MCl}$ population, patients with only $\mathrm{A}$ biomarker positive $(\mathrm{A}+\mathrm{T}-\mathrm{N}-)$ clinically progress at a rate similar to that of $\mathrm{A}-\mathrm{T}-\mathrm{N}-$ profile. Our findings suggested that the isolated amyloid abnormality $(\mathrm{A}+)$ indicated a relatively stable state rather than a sign of accelerated cognitive decline. In fact, amyloid deposition is the initial event of AD-related pathophysiologic change[35], which can last for 5-10 years or longer before the onset of dementia symptoms[36]. On the basis of abnormal $A \beta$ plaques, each added biomarker of $\mathrm{T}$ or $\mathrm{N}$ will increase the recent progress rate of $\mathrm{MCl}$ patients. The progression rate was from less than $30 \%$ in $\mathrm{A}+\mathrm{T}-\mathrm{N}$ - to more than $60 \%$ in $\mathrm{A}+\mathrm{T}+\mathrm{N}-$ or $\mathrm{A}+\mathrm{T}-\mathrm{N}+$, and finally $92 \%$ in $\mathrm{A}+\mathrm{T}+\mathrm{N}+$. Interestingly, similar risk of progression was observed between $\mathrm{A}+\mathrm{T}+\mathrm{N}-$ and $\mathrm{A}+\mathrm{T}-\mathrm{N}+$, suggesting that $\mathrm{T}$ and $\mathrm{N}$ may play equal roles in the progression prediction. A previous study has suggested that $\mathrm{A}+\mathrm{T}+\mathrm{N}-$ and $\mathrm{A}+\mathrm{T}+\mathrm{N}+$ should be combined into a single group to simplify the ATN scheme because of their similar baseline characteristics[9].

However, in this study we found significant differences in baseline status and prognosis between the two groups. Patients with $A+T+N+$ have higher risk of cognitive progress, so special attention should be paid and timely intervention should be given.

Suspected non-Alzheimer's disease pathophysiology (SNAP) is considered to be an important category, which refers to individuals without excessive amyloid deposition $(A-)$, but with tau pathology $(T+)$ and / or neurodegenerative disease $(N+)$. SNAP does not represent preclinical $A D$, but includes one or more neuropathological processes or diseases other than AD[37]. SNAP is common in both clinically asymptomatic and cognitively impaired individuals[38, 39]. In our study, the presence of SNAP was relatively consistent across different clinical groups ( $\mathrm{HC} 24.5 \%, \mathrm{MCl} 22.3 \%$, and $\mathrm{AD} 21.1 \%)$. During the follow-up, we found that $\mathrm{N}$ biomarker evaluated by radiomics features was sensitive at predicting recent cognitive decline for SNAP MCl. In fact, a variety of non-AD processes such as TDP-4, hippocampal sclerosis, or cerebrovascular disease are likely to contribute to neurodegeneration in these individuals[40, 41]. Neuronal loss and atrophy are common features of these diseases. In contrast, most MCl patients with A - T + N-characteristics in SNAP showed clinical stability, indicating that single CSF p-tau abnormal does not lead to further cognitive decline. The possible reason is that p-tau in SNAP mostly reflects agerelated neurofilament tangle pathology rather than $A D$ related neuronal degeneration.

\section{Limitations}

there were several limitations to our study. First of all, due to the strict inclusion conditions, the sample size of this study was not large enough, leading to the insufficient numbers in some ATN profiles. Secondly, considering the limitations of clinical application, biomarkers for PET were not included. Finally, our analysis was limited to observing the relationship between baseline biomarker status and progression risk of $\mathrm{MCl}$ patients, and no longitudinal analysis was 
performed. Future research should break through these limitations and analyze the relationship between the dynamic changes of these indicators and the progress of cognitive impairment by using large samples.

\section{Conclusions}

In this study, we proposed a new radiomics method to assess neurodegeneration accurately and ascertained the optimal CSF and MRI A/T/N biomarkers for the discrimination of $\mathrm{HC}$ and $\mathrm{AD}$. For $\mathrm{MCl}$ patients of the Alzheimer continuum, isolated $\mathrm{A}+$ was indicator of cognitive stability, while the abnormality of $\mathrm{T}$ and $\mathrm{N}$, respectively or simultaneously, indicated the high risk of progression. For MCl patients of SNAP, isolated T + indicated the cognitive stability, while the appearance of $\mathrm{N}+$ indicated the high risk of progression.

\section{Abbreviations}

ATN: amyloid/tau/neurodegeneration; CSF: Cerebrospinal fluid; ADNI: Alzheimer's Disease Neuroimaging Initiative; MCl: mild cognitive impairment individuals; HC: healthy control; AD: Alzheimer' Disease; A $\beta$ : $\beta$-amyloid; p-tau: Phosphorylated tau; t-tau: Total tau; AUC: Area under the curve; ROC: Receiver operating characteristic; SNAP: Suspected non-AD pathophysiology; MMSE: Mini-Mental State Examination; CDR: Clinical Dementia Rating; FAQ: Functional Activities Questionnaire; GDS: Geriatric Depression Scale; RAVLT: Rey Auditory Verbal Learning Test; AFT: Animal Fluency Test; ADAS-Cog: Alzheimer's Disease Assessment Scale-Cognitive subscale; MRI: Magnetic resonance image; 3D MPRAGE: Three-dimensional magnetization prepared rapid gradient echo; LASSO: Least absolute shrinkage and selection operator

\section{Declarations}

\section{Acknowledgements}

We are grateful for the research volunteers, their families, and the investigators at the ADNI database. ADNI is funded by the National Institute on Aging, the National Institute of Biomedical Imaging and Bioengineering, and through generous contributions from the following: AbbVie, Alzheimer's Association; Alzheimer's Drug Discovery Foundation; Araclon Biotech; BioClinica, Inc.; Biogen; Bristol Myers Squibb Company; CereSpir, Inc.; Cogstate; Eisai Inc.; Elan Pharmaceuticals, Inc.; Eli Lilly and Company; Eurolmmun; F. Hoffmann-La Roche Ltd. and its affiliated company Genentech, Inc.; Fujirebio; GE Healthcare; IXICO Ltd.; Janssen Alzheimer Immunotherapy Research \& Development, LLC.; Johnson \& Johnson Pharmaceutical Research \& Development LLC.; Lumosity; Lundbeck; Merck \& Co., Inc.; Meso Scale Diagnostics, LLC.; NeuroRx Research; Neurotrack Technologies; Novartis Pharmaceuticals Corporation; Pfizer Inc.; Piramal Imaging; Servier; Takeda Pharmaceutical Company; and Transition Therapeutics. The Canadian Institutes of Health Research is providing funds to support ADNI clinical sites in Canada. Private sector contributions are facilitated by the Foundation for the National Institutes of Health (www.fnih.org). The grantee organization is the Northern California Institute for Research and Education, and the study is coordinated by the Alzheimer's Therapeutic Research Institute at the University of Southern California. ADNI data are disseminated by the Laboratory for Neuro Imaging at the University of Southern California.

Partial data used in the preparation of this article were obtained from the Alzheimer's Disease Neuroimaging Initiative (ADNI) database (adni.loni.usc.edu). As such, the investigators within the ADNI contributed to the design and implementation of ADNI and/or provided data but did not participate in the analysis or writing of this report. A complete listing of ADNI investigators can be found at http://adni.loni.usc.edu/wpcontent/uploads/how_to_apply/\%20ADNI_Acknowledgement_List.pdf.

\section{Authors' contributions}

CML, RS and XJW contributed conceived and designed the study, analyzed and interpreted the data, and drafted and revised the manuscript. RS, XJW and HL did the statistical analysis, and prepared all the figures. DJG interpreted the data and revised of the manuscript. All authors took part in revising the manuscript for content and approved the final version. Data used in this study were obtained from the ADNI database. The investigators within the ADNI were uninvolved in the data analysis or writing of this report.

\section{Funding}

This work was supported by the Chongqing Science and Health Joint Medical Research Project of China, Grant/Award Number: 2018ZDXM005; the Chongqing Natural Science Foundation, Grant/Award Number: cstc2020jcyj-msxmX0044; the Kuanren Talents Program of the Second Affiliated Hospital of Chongqing Medical University, Grant/Award Number: 2020-7. The funding body had no role in the design of the study and collection, analysis, interpretation of data, and writing of the manuscript.

\section{Availability of data and materials}

The dataset generated and analyzed in the current study is available from the corresponding author on reasonable request.

\section{Ethics approval and consent to participate}

The ADNI study was approved by an ethics standards committee on human experimentation at each institution. Written informed consent was obtained from all participants.

\section{Consent for publication}

Not applicable. 


\section{Competing interests}

The authors declare that they have no competing interests.

\section{Author details}

${ }^{1}$ Department of Radiology, the Second Affiliated Hospital of Chongqing Medical University, No. 74 Linjiang Rd, Yuzhong District, Chongqing 400010, China. ${ }^{2}$ GE Healthcare, Shanghai, China, ${ }^{3}$ Department of Radiology, the Emergency Medical Center of Chongqing, No. 1 Jiankang Rd, Yuzhong District, Chongqing 400000, China.

\section{References}

1. Hou Y, Dan X, Babbar M, Wei Y, Hasselbalch SG, Croteau DL et al. Ageing as a risk factor for neurodegenerative disease. Nat Rev Neurol. 2019; 15(10):565581.

2. 2020 Alzheimer's disease facts and figures. Alzheimers Dement. 2020; 16(3):391-460.

3. Ngandu T, Lehtisalo J, Solomon A, Levälahti E, Ahtiluoto S, Antikainen R et al. A 2 year multidomain intervention of diet, exercise, cognitive training, and vascular risk monitoring versus control to prevent cognitive decline in at-risk elderly people (FINGER): a randomised controlled trial. Lancet. 2015; 385(9984):2255-2263.

4. Crous-Bou M, Minguillón C, Gramunt N, Molinuevo JL. Alzheimer's disease prevention: from risk factors to early intervention. Alzheimers Res Ther. 2017; $9(1): 71$.

5. Jack CR, Bennett DA, Blennow K, Carrillo MC, Dunn B, Haeberlein SB et al. NIA-AA Research Framework: Toward a biological definition of Alzheimer's disease. Alzheimers Dement. 2018; 14(4):535-562.

6. Hwang J, Jeong J, Yoon S, Park K, Kim E-J, Yoon B et al. Clinical and Biomarker Characteristics According to Clinical Spectrum of Alzheimer's Disease $(A D)$ in the Validation Cohort of Korean Brain Aging Study for the Early Diagnosis and Prediction of AD. J Clin Med. 2019; 8(3).

7. Devanarayan P, Devanarayan V, Llano DA. Identification of a Simple and Novel Cut-Point Based Cerebrospinal Fluid and MRI Signature for Predicting Alzheimer's Disease Progression that Reinforces the 2018 NIA-AA Research Framework. J Alzheimers Dis. 2019; 68(2):537-550.

8. Illán-Gala I, Pegueroles J, Montal V, Vilaplana E, Carmona-Iragui M, Alcolea D et al. Challenges associated with biomarker-based classification systems for Alzheimer's disease. Alzheimers Dement (Amst). 2018; 10(1):346-357.

9. Altomare D, de Wilde A, Ossenkoppele R, Pelkmans W, Bouwman F, Groot C et al. Applying the ATN scheme in a memory clinic population. Neurology. 2019; 93(17):e1635-e1646.

10. Ebenau JL, Timmers T, Wesselman LMP, Verberk IMW, Verfaillie SCJ, Slot RER et al. ATN classification and clinical progression in subjective cognitive decline. Neurology. 2020; 95(1):e46-e58.

11. Calvin CM, de Boer C, Raymont V, Gallacher J, Koychev I. Prediction of Alzheimer's disease biomarker status defined by the 'ATN framework' among cognitively healthy individuals: results from the EPAD longitudinal cohort study. Alzheimers Res Ther. 2020; 12(1):143.

12. Lehmann M, Rohrer JD, Clarkson MJ, Ridgway GR, Scahill RI, Modat M et al. Reduced Cortical Thickness in the Posterior Cingulate Gyrus is Characteristic of Both Typical and Atypical Alzheimer's Disease. J Alzheimers Dis. 2010; 20(2):587-598.

13. de Jong LW, van der Hiele K, Veer IM, Houwing JJ, Westendorp RGJ, Bollen ELEM et al. Strongly reduced volumes of putamen and thalamus in Alzheimer's disease: an MRI study. Brain. 2008; 131(12):3277-3285.

14. Cho H, Kim J-H, Kim C, Ye BS, Kim HJ, Yoon CW et al. Shape Changes of the Basal Ganglia and Thalamus in Alzheimer's Disease: A Three-Year Longitudinal Study. J Alzheimers Dis. 2014; 40(2):285-295.

15. Langa KM, Levine DA. The Diagnosis and Management of Mild Cognitive Impairment. Jama. 2014; 312(23):2551-2561.

16. Vos SJB, Verhey F, Frölich L, Kornhuber J, Wiltfang J, Maier W et al. Prevalence and prognosis of Alzheimer's disease at the mild cognitive impairment stage. Brain. 2015; 138(5):1327-1338.

17. Ekman U, Ferreira D, Westman E. The A/T/N biomarker scheme and patterns of brain atrophy assessed in mild cognitive impairment. Sci Rep. 2018; $8(1): 8431$.

18. McKhann G, Drachman D, Folstein M, Katzman R, Price D, Stadlan EM. Clinical diagnosis of Alzheimer's disease: Report of the NINCDS-ADRDA Work Group* under the auspices of Department of Health and Human Services Task Force on Alzheimer's Disease. Neurology. 1984; 34(7):939-939.

19. Dubois B, Feldman HH, Jacova C, Dekosky ST, Barberger-Gateau P, Cummings $\mathrm{J}$ et al. Research criteria for the diagnosis of Alzheimer's disease: revising the NINCDS-ADRDA criteria. Lancet Neurol. 2007; 6(8):734-746.

20. Terry RD, Masliah E, Salmon DP, Butters N, DeTeresa R, Hill R et al. Physical basis of cognitive alterations in alzheimer's disease: Synapse loss is the major correlate of cognitive impairment. Ann Neurol. 1991; 30(4):572-580.

21. Sørensen L, Igel C, Liv Hansen N, Osler M, Lauritzen M, Rostrup E et al. Early detection of Alzheimer's disease using MRI hippocampal texture. Hum Brain Mapp. 2016; 37(3):1148-1161.

22. Luk CC, Ishaque A, Khan M, Ta D, Chenji S, Yang YH et al. Alzheimer's disease: 3-Dimensional MRI texture for prediction of conversion from mild cognitive impairment. Alzheimers Dement (Amst). 2018; 10(1):755-763.

23. Huang K, Lin Y, Yang L, Wang Y, Cai S, Pang L et al. A multipredictor model to predict the conversion of mild cognitive impairment to Alzheimer's disease by using a predictive nomogram. Neuropsychopharmacology. 2019; 45(2):358-366. 
24. Shinde S, Prasad S, Saboo Y, Kaushick R, Saini J, Pal PK et al. Predictive markers for Parkinson's disease using deep neural nets on neuromelanin sensitive MRI. Neuroimage Clin. 2019; 22:101748.

25. Li G, Zhai G, Zhao X, An H, Spincemaille P, Gillen KM et al. 3D texture analyses within the substantia nigra of Parkinson's disease patients on quantitative susceptibility maps and R2* maps. Neurolmage. 2019; 188:465-472.

26. Geroldi C, Rossi R, Calvagna C, Testa C, Bresciani L, Binetti G et al. Medial temporal atrophy but not memory deficit predicts progression to dementia in patients with mild cognitive impairment. Journal Neurol Neurosurg Psychiatry. 2006; 77(11):1219-1222.

27. Fennema-Notestine C, Hagler DJ, McEvoy LK, Fleisher AS, Wu EH, Karow DS et al. Structural MRI biomarkers for preclinical and mild Alzheimer's disease. Hum Brain Mapp. 2009; 30(10):3238-3253.

28. Whitwell JL, Przybelski SA, Weigand SD, Knopman DS, Boeve BF, Petersen RC et al. 3D maps from multiple MRI illustrate changing atrophy patterns as subjects progress from mild cognitive impairment to Alzheimer's disease. Brain. 2007; 130(7):1777-1786.

29. Masters C, Bateman R, Blennow K, Rowe C, Sperling R, Cummings JJNrDp. Alzheimer's disease. Nat Rev Dis Primers. 2015 ; 1:15056.

30. Jacobs HIL, Van Boxtel MPJ, Jolles J, Verhey FRJ, Uylings HBM. Parietal cortex matters in Alzheimer's disease: An overview of structural, functional and metabolic findings. Neurosci Biobehav Rev. 2012; 36(1):297-309.

31. Hoř́nek D, Varjassyová A, Hort J. Magnetic resonance analysis of amygdalar volume in Alzheimer's disease. Curr Opin Psychiatry. 2007; 20 (3):273-277.

32. Tang X, Holland D, Dale AM, Miller MI, Adamson M. APOE Affects the Volume and Shape of the Amygdala and the Hippocampus in Mild Cognitive Impairment and Alzheimer's Disease: Age Matters. J Alzheimers Dis. 2015; 47(3):645-660.

33. Blennow K, Vanmechelen E, Hampel H. CSF Total tau, A 42 and Phosphorylated tau Protein as Biomarkers for Alzheimer's Disease. Mol Neurobiol. 2001; 24(1-3):087-098.

34. Rosa MI, Perucchi J, Medeiros LR, Fernandes B, Fernandes dos Reis ME, Silva BR. Accuracy of Cerebrospinal Fluid Aß1-42 for Alzheimer's Disease Diagnosis: A Systematic Review and Meta-Analysis. J Alzheimers Dis. 2014; 40(2):443-454.

35. Golde TE, Eckman CB, Younkin SG. Biochemical detection of A isoforms: implications for pathogenesis, diagnosis, and treatment of Alzheimer's disease. Biochim Biophys Acta. 2000; 1502(1):172-187.

36. Buchhave P, Minthon L, Zetterberg H, Wallin AK, Blennow K, Hansson O. Cerebrospinal fluid levels of $\beta$-amyloid 1-42, but not of tau, are fully changed already 5 to 10 years before the onset of Alzheimer dementia. Arch Gen psychiatry. 2012; 69(1):98-106.

37. Jack CR, Knopman DS, Weigand SD, Wiste HJ, Vemuri P, Lowe V et al. An operational approach to National Institute on Aging-Alzheimer's Association criteria for preclinical Alzheimer disease. Ann Neurol. 2012; 71(6):765-775.

38. Soldan A, Pettigrew C, Fagan AM, Schindler SE, Moghekar A, Fowler C et al. ATN profiles among cognitively normal individuals and longitudinal cognitive outcomes. Neurology. 2019; 92(14):e1567-e1579.

39. Grøntvedt GR, Lauridsen C, Berge G, White LR, Salvesen $\emptyset$, Bråthen G et al. The Amyloid, Tau, and Neurodegeneration (A/T/N) Classification Applied to a Clinical Research Cohort with Long-Term Follow-Up. J Alzheimers Dis. 2020; 74(3):829-837.

40. Sonnen JA, Larson EB, Crane PK, Haneuse S, Li G, Schellenberg GD et al. Pathological correlates of dementia in a longitudinal, population-based sample of aging. Ann Neurol. 2007; 62(4):406-413.

41. James BD, Wilson RS, Boyle PA, Trojanowski JQ, Bennett DA, Schneider JA. TDP-43 stage, mixed pathologies, and clinical Alzheimer's-type dementia. Brain. 2016; 139(11):2983-2993.

\section{Figures}


A

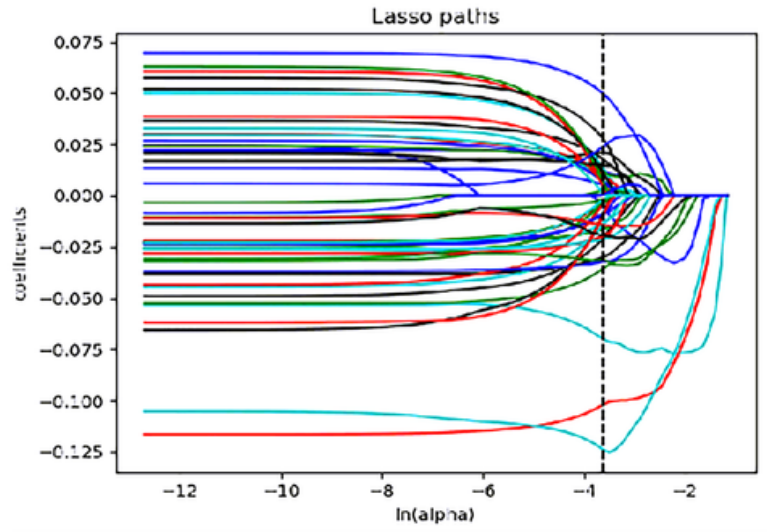

C

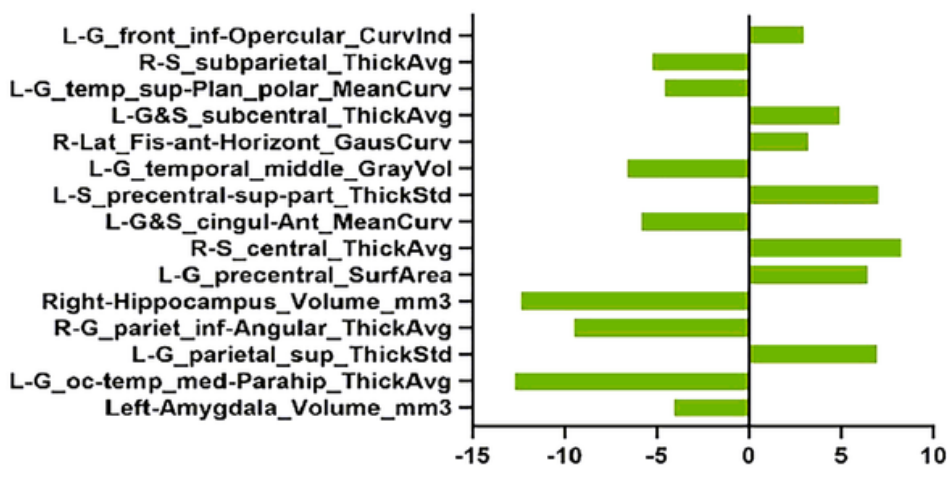

B

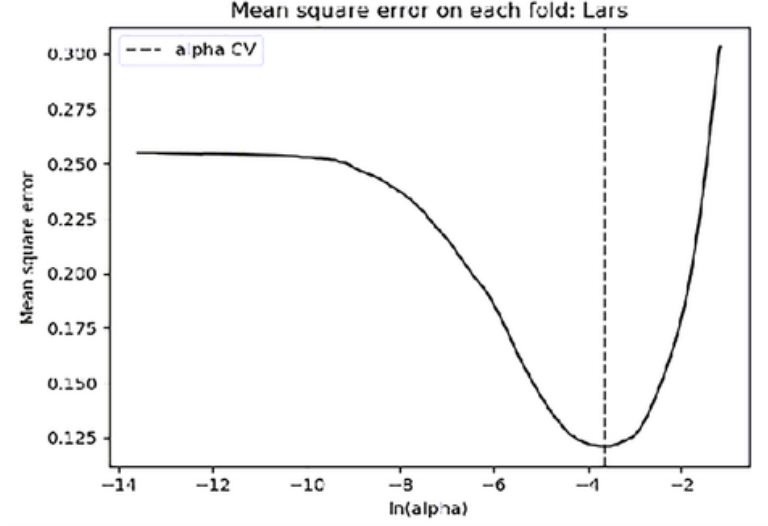

D

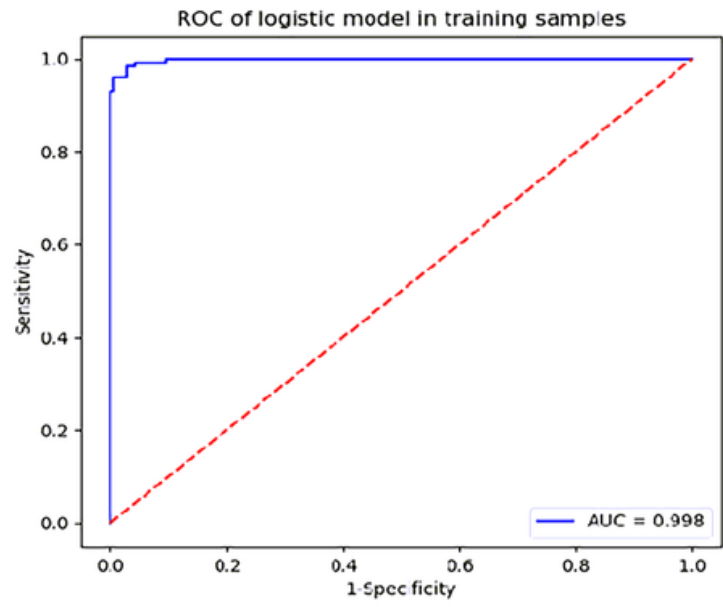

Figure 1

Results of radiomics feature selection and radiomics model construction. A: LASSO coefficient analysis of the radiomics features. B: Mean square error of each fold. C: A histogram displays the 15 optimal radiomics features for AD diagnosis from the $\mathrm{HC}$ and their coefficients. D: The ROC curve of the radiomics model for discrimination between AD and HC. LASSO, least absolute shrinkage and selection operator; AD, Alzheimer' disease; HC, healthy control; ROC, receiver operating characteristic. 
A

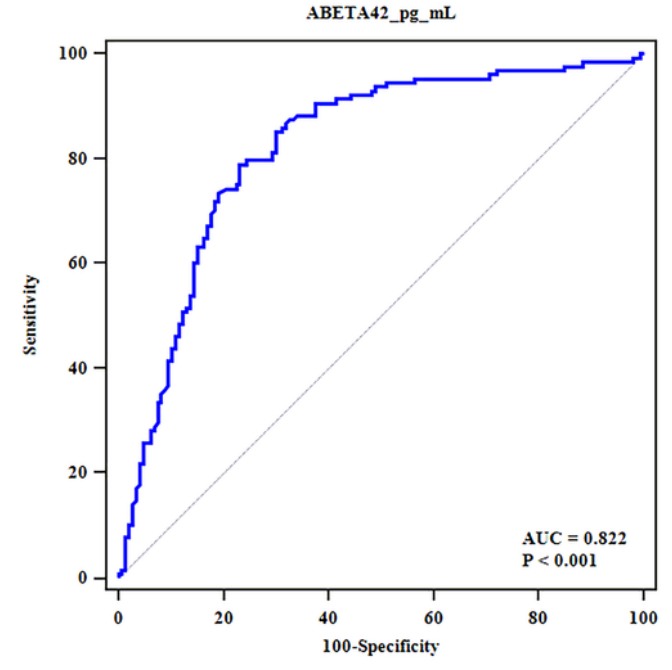

C

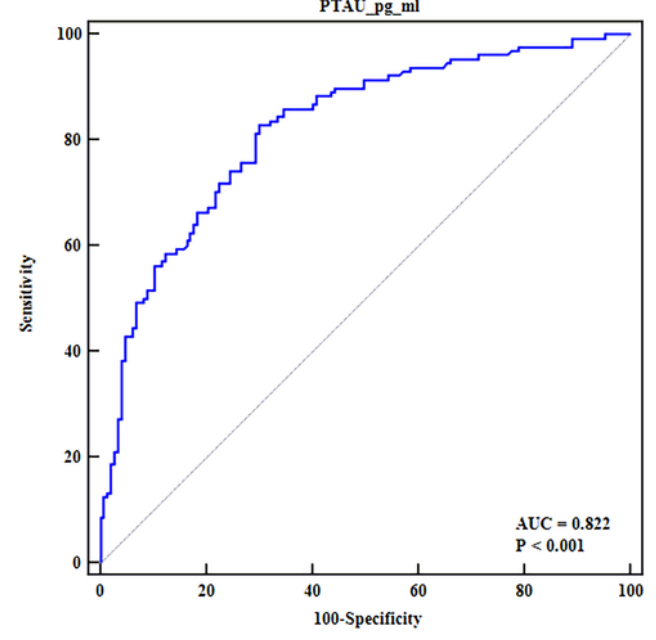

B

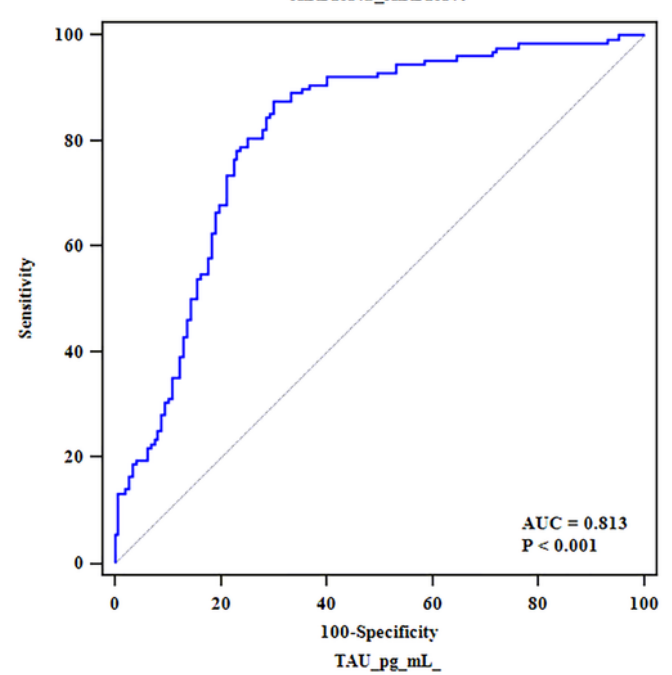

D

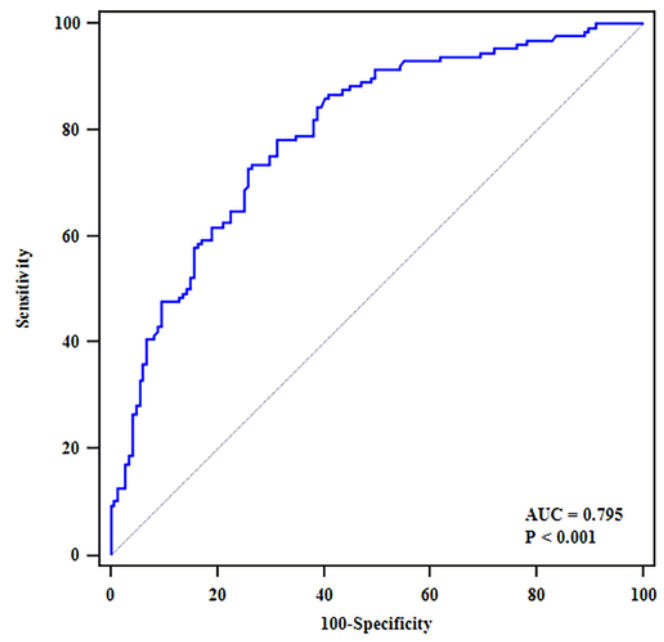

\section{Figure 2}

ROC curves of the CSF biomarkers for discrimination between Alzheimer' disease and healthy controls. A: ROC curve of CSF A 42 . B: ROC curve of CSF Aß42/Aß40. C: ROC curve of CSF p-tau. D: ROC curve of CSF t-tau. ROC, receiver operating characteristic. CSF, cerebrospinal fluid. 


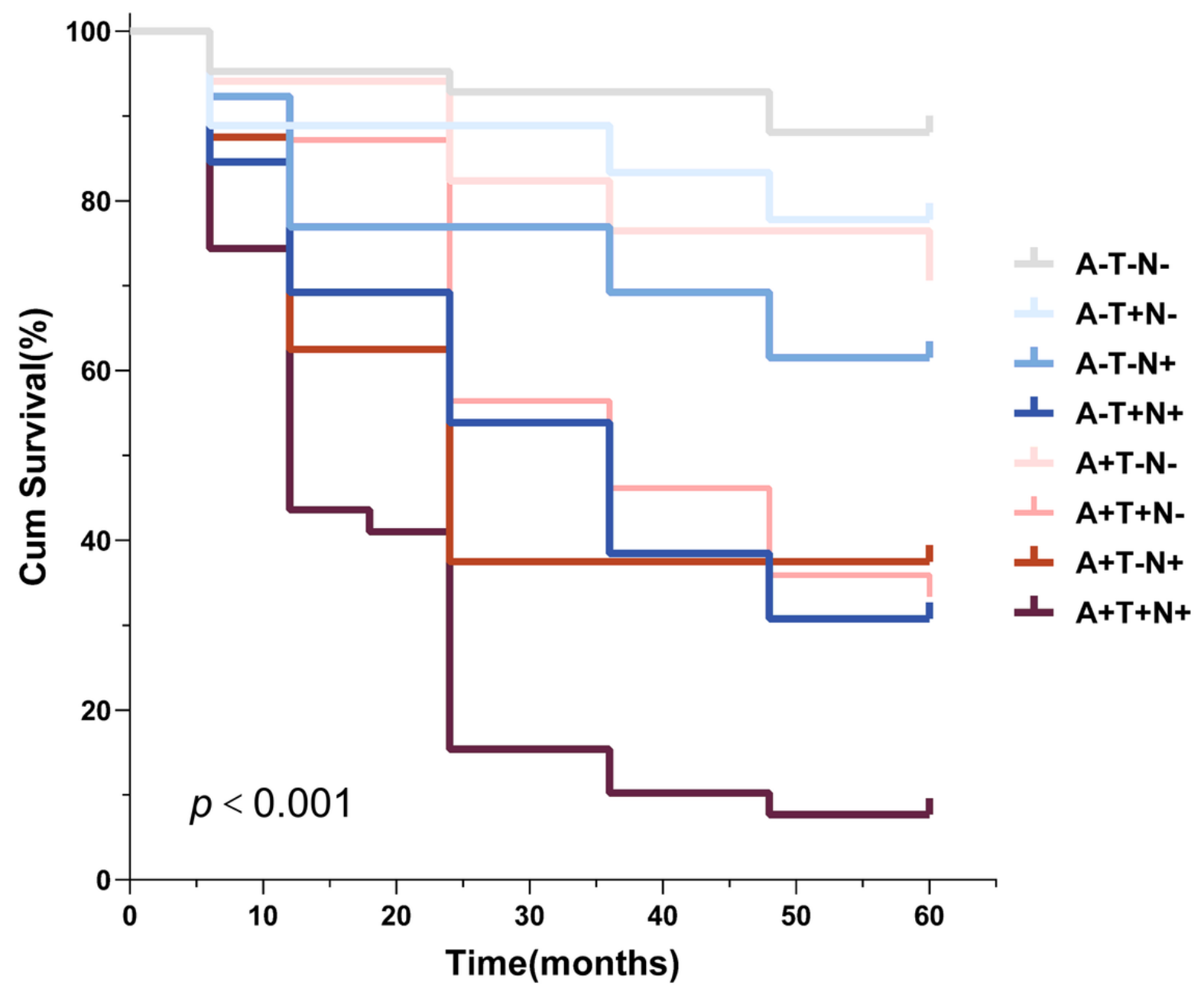

Figure 3

Kaplan-Meier curves illustrated the progression to dementia in 8 ATN profiles of mild cognitive impairment.

\section{Supplementary Files}

This is a list of supplementary files associated with this preprint. Click to download.

- Additionalfile1.docx

- Additionalfile2.docx

- Additionalfile3.docx

- Additionalfile4.docx

- Additionalfile5.docx 